\title{
Mitochondrial destiny in type 2 diabetes: the effects of oxidative stress on the dynamics and biogenesis of mitochondria
}

\author{
Daria Skuratovskaia ${ }^{\text {Corresp., } 1}{ }^{1}$, Alexandra Komar ${ }^{1}$, Maria Vulf $^{1}$, Larisa Litvinova ${ }^{1}$ \\ 1 Immanuel Kant Baltic Federal University, Kaliningrad, Russian Federation \\ Corresponding Author: Daria Skuratovskaia \\ Email address: dariask@list.ru
}

Background. One reason for the development of insulin resistance is the chronic inflammation in obesity. Materials \& Methods. Scientific articles in the field of knowledge on the involvement of mitochondria and mitochondrial DNA (mtDNA) in obesity and type 2 diabetes were analyzed. Results. Oxidative stress developed during obesity contributes to the formation of peroxynitrite, which causes cytochrome C-related damage in the mitochondrial electron transfer chain and increases the production of reactive oxygen species (ROS), which is associated with the development of type 2 diabetes. Oxidative stress contributes to the nuclease activity of the mitochondrial matrix, which leads to the accumulation of cleaved fragments and an increase in heteroplasmy. Mitochondrial dysfunction and mtDNA variations during insulin resistance may be connected with a change in ATP levels, generation of ROS, mitochondrial division/fusion and mitophagy. This review discusses the main role of mitochondria in the development of insulin resistance, which leads to pathological processes in insulin-dependent tissues, and considers potential therapeutic directions based on the modulation of mitochondrial biogenesis. In this regard, the development of drugs aimed at the regulation of these processes is gaining attention. Conclusion. Changes in the mtDNA copy number can help to protect mitochondria from severe damage during conditions of increased oxidative stress. Mitochondrial proteome studies are conducted to search for potential therapeutic targets. The use of mitochondrial peptides encoded by mtDNA also represents a promising new approach to therapy 
1 Mitochondrial destiny in type 2 diabetes: the effects of

2 oxidative stress on the dynamics and biogenesis of

3 mitochondria

4

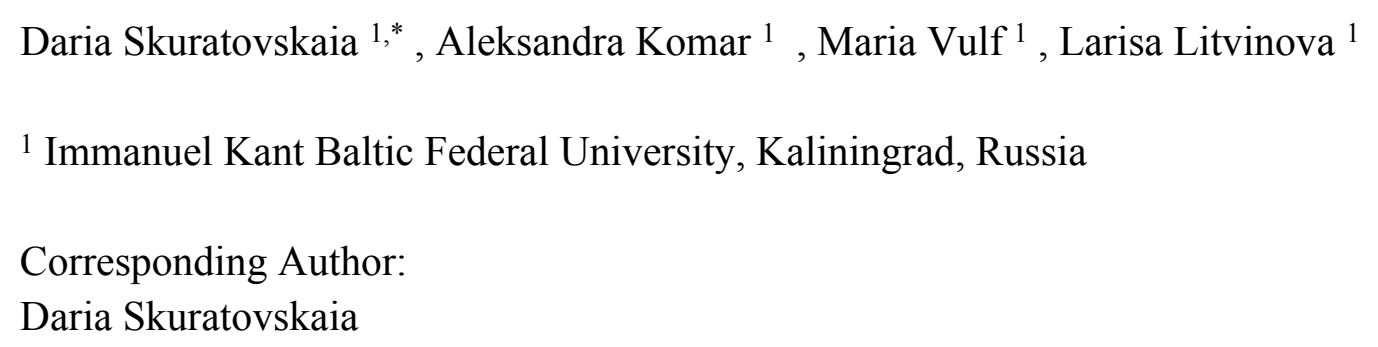

\section{Abstract}

Background. One reason for the development of insulin resistance is the chronic inflammation in obesity.

Materials \& Methods. Scientific articles in the field of knowledge on the involvement of mitochondria and mitochondrial DNA (mtDNA) in obesity and type 2 diabetes were analyzed. Results. Oxidative stress developed during obesity contributes to the formation of peroxynitrite, which causes cytochrome C-related damage in the mitochondrial electron transfer chain (ETC) and increases the production of reactive oxygen species (ROS), which is associated with the development of type 2 diabetes. Oxidative stress contributes to the nuclease activity of the mitochondrial matrix, which leads to the accumulation of cleaved fragments and an increase in heteroplasmy. Mitochondrial dysfunction and mtDNA variations during insulin resistance may be connected with a change in ATP levels, generation of ROS, mitochondrial division/fusion and mitophagy. This review discusses the main role of mitochondria in the development of insulin resistance, which leads to pathological processes in insulin-dependent tissues, and considers potential therapeutic directions based on the modulation of mitochondrial biogenesis. In this regard, the development of drugs aimed at the regulation of these processes is gaining attention. Conclusion. Changes in the mtDNA copy number can help to protect mitochondria from severe damage during conditions of increased oxidative stress. Mitochondrial proteome studies are conducted to search for potential therapeutic targets. The use of mitochondrial peptides encoded by mtDNA also represents a promising new approach to therapy.

\section{Introduction}

Diets that are high in fat and/or sucrose can lead to insulin resistance (IR) and are often associated with mitochondrial dysfunction. Both types of diet can be considered extreme and lead to a significant change in the normal balance between substrates of cellular intermediary metabolism [1]. As a rule, this is also associated with excess substrate in a particular diet, that is, 
40 fatty acids or sucrose (glucose plus fructose), creating an overload in the cell. Therefore, it is 41 likely that this type of altered metabolic condition affects the normal regulatory mechanisms of 42 cells, particularly in insulin-dependent tissues, such as adipose tissue, muscle, and liver.

43 Mitochondria are double-membrane organelles. Process oxidative phosphorylation (OXPHOS)

44 enables ATP generation in mitochondria. Membrane electrochemical gradient stimulates the 45 synthesis of OXPHOS and ATP. This gradient includes electrons are transferred to nicotinamide 46 adenine dinucleotide (NAD+) and flavin adenine dinucleotide (FAD), and NADH and FADH2

47 are produced. These molecules are formed as a result of the course of the tricarboxylic acid 48 (TCA) cycle, which is generated by the oxidation process of acetyl coenzyme A (acetyl-CoA). In 49 turn, acetyl-CoA is formed by the react oxidation of fatty acids and pyruvate [2]. Electrons are

50

51

52

53

54

55

56

57

58

59

60

61

62

63

64

65

66

67

68

69

70

\section{1}

72

73

74

75

76

77

78 accepted and transported via components of the electron transfer chain (ETC) in the inner mitochondrial membrane. The electron transfer along the ETC is associated with proton transfer through the inner membrane. This process establishes an electrochemical gradient, which is necessary to regulate the synthesis of ATP by the ATP synthase, FoF1-ATPase [3].

Mitochondria in adipocytes can play a significant role in the regulation of energy homeostasis of the whole body, in controlling sensitivity to insulin and glucose metabolism. Mitochondria are a regulator of lipolysis in adipocytes and the main source of ATP in cells. Mitochondria are key organelles that control the physiological role of adipocytes: differentiation, lipid homeostasis, insulin sensitivity, oxidative ability, adaptive thermogenesis. Disruption of the functioning of mitochondria in insulin-dependent tissues leads to an energy crisis, which underlies the formation of IR [4].

Given the potentially important role of mitochondrial dysfunction in the pathogenesis of many diseases and the process of ageing, an understanding of the molecular mechanisms responsible for mitochondrial biogenesis and function could lead to important new therapeutic targets. Systematization of data on this topic allows you to get an overview of the current state of research in this area as a whole, rather than detailed knowledge of the area in question, as well as get links to the most useful primary sources

This review provides a solid starting point for all community members interested in a particular research topic and is a source of information for practitioners who seek the latest evidence that can guide them in their decision making and work.

\section{Survey Methodology}

We have provided comprehensive and objective coverage of topics by synthesizing existing literature and identifying knowledge gaps in empirical research. We used the search engines PubMed, MedLine, ClinicalTrials, Kegg data base.

We searched for keywords and word combinations: mtDNA, deletion mtDNA, mitochondria, oxidative stress, obesity, type 2 diabetes, peptide, proteome, genome, MOTS-C, TNF, IL, inflammation, glucotoxicity and lipotoxicity, biogenesis, mitophagy, dynamics of mitochondria, ROS, autophagy, mitochondria-dependent apoptosis. 
79 We selected articles on the basis of certain eligibility criteria - the quality of the work performed,

80 based on the ratings of journals, and this is a search depth of research articles of no more than 5 81 years.

82

\section{Factors contributing to the development of mitochondrial dysfunction and insulin resistance}

The mechanisms for the development of IR have been studied extensively; however, consensus on a single mechanism has not yet been reached. It remains unclear whether mitochondrial dysfunction is a result of IR or whether IR itself causes mitochondrial dysfunction. One generally accepted theory for the development of IR suggests that inflammation contributes to the increased production of reactive oxygen species reactive oxygen species (ROS). In obesity, adipocytes undergo hypertrophy, promoting the release of free fatty acids during lipolysis and the development of hypoxia in adipose tissue [4,5]. Immune cells and macrophages are then recruited to adipose tissue by inflammatory mediators [5]. The necrotic adipocytes are phagocytosed by macrophages, which produce chemokines and pro-inflammatory mediators (Fig, 1) [5, 6].

Transcription factors involved in the pro-inflammatory pathways, Nuclear factor kappa B (NF$\mathrm{kB}$ ), signal transducer and activator of transcription (STAT), and Activator protein (AP-1), are activated, triggering subsequent signalling cascades that mediate the expression of proteins, which then inhibit the insulin signalling pathway, contributing to the development of IR $[4,6]$. Signalling molecules can interact with effectors on the surface of mitochondria [4, 7]. Excessive peroxynitrite formation causes mitochondrial ETC to release cytochrome $\mathrm{C}$ and increases the production of ROS (Fig. 1) [8], which are associated with the development of type 2 diabetes [7]. For example, the tumour necrosis factor-alfa (TNF- $\alpha$ ) induces mitochondrial dysfunction, decreasing the activity of complex III in ETC, increasing the production of ROS, and causing damage to mitochondrial DNA (mtDNA). Mitochondrial superoxide anion is a precursor of most ROS and a mediator in the reaction of oxidative stress. Superoxide dismutase leads to the formation of hydrogen peroxide and a hydroxyl radical. There radicals causing damage to various mitochondrial and cellular components. Such damage in the mitochondria can lead to a decrease in the affinity of mitochondrial proteins for substrates or coenzymes [7].

There is no doubt that genetic mutations in mtDNA, which can be either innate or acquired, are associated with the development of IR; numerous studies have shown the relationship between polymorphisms and the development of metabolic disorders. For example, dysfunctional mtDNA reduces the efficiency of OXPHOS and ATP synthesis [9]. Under oxidative stress, mitochondria generate superoxide, hydrogen peroxide, and hydroxyl radicals, thereby contributing to protein, DNA, RNA, and lipid damage [10,11].

Oxidative stress contributes to the nuclease activity of the mitochondrial matrix, which leads to the accumulation of cleaved fragments and an increased level of heteroplasmy. Under oxidative stress, mtDNA is damaged by deletions $[12,13]$. Patients with mt DNA mutations are 
119 characterized by decreased functioning of pancreatic beta cells and decreased glucose-stimulated 120 insulin secretion $[14,15]$.

121 Ye J. (2013) proposed the relationship between energy metabolism in mitochondria and IR.

122 According to this hypothesis, IR is the result of excess energy in cells [16], and a decrease in the

123 functional activity of mitochondria is considered to be an adaptive mechanism for the soft

124 separation of the ETC, which protects mitochondria from the vicious cycle of ROS production.

125 The author suggested that this mechanism offers a promising approach to the treatment of IR

126 [16].

127 The effects of certain drugs for the treatment of type 2 diabetes prove this hypothesis. Among the

128 mechanisms of action of these drugs that lead to improved insulin sensitivity, is the suppression

129 of ATP generation in mitochondria $[9,16]$. In addition, effective weight loss treatments,

130 exercise, and a low-calorie diet are effective treatments for insulin-dependent ATP in insulin-

131 sensitive tissues [16]. The new concept assumes the existence of a unified cellular and molecular

132 mechanism of IR in obesity.

133 Therefore, mitochondria play a key role in the pathogenesis of IR by regulating lipolysis in

134 adipocytes and serving as the main sources of ATP in cells (Fig. 1) [17]. Impaired mitochondrial

135 function in insulin-dependent tissues leads to an energy crisis that underlies the development of

136 IR $[9,18]$.

137

138

139

140

141

142

143

144

145

146

147

148

149

150

151

152

153

154

155

156

157

158

\subsection{Disturbances of mitochondrial fission and fusion during oxidative stress}

Mitochondrial fission and fusion play a crucial role in maintaining functional mitochondria when cells experience metabolic or environmental stress. Fusion helps to alleviate stress by combining the contents of partially damaged mitochondria, whereas fission is necessary to create new mitochondria while also contributing to quality control by enabling the removal of damaged mitochondria and promoting apoptosis during high levels of cellular stress [19]. Mitochondrial biogenesis is mainly regulated by several key transcription factors, such as peroxisome proliferator-activated receptor gamma coactivator 1-alpha (PGC-1 $\alpha$ ), which regulates the generation of mitochondrial proteins. Mitochondrial dynamics are regulated mainly by a family of dynamin-like GTPases, of which dynamin-related protein 1 (Drp1) is one member. Drp1 is mostly localized to the cytoplasm but can translocate to mitochondria, where it participates in the division of the outer mitochondrial membrane [20]. Two other members of this family are mitofusins (Mfn 1 and Mfn2), and optic atrophy type-1 (OPA1). Mfn1 and Mfn2 control the outer mitochondrial membrane, while OPA1 is located in and controls the inner membrane [21].

Normally, to maintain energy balance, mitochondria use a mechanism of adaptation to the metabolic needs of the cell - division and fusion. This process is still poorly understood; however its relationship with mitochondrial bioenergy is undeniable. Mitochondrial transcription factor A (TFAM), one of the critical regulators of mtDNA transcription, is associated with changes in mitochondrial fusion and mtDNA replication [22]. Thus, in patients with type 2 diabetes, an 
159 increase in the level of transcription of the TFAM gene and a decrease in its protein level in vitro 160 models were noted [22].

161 Balanced control of fusion and division is important for mitochondrial bioenergy [23].

162 Mitochondrial fusion processes are induced when optimization of mitochondrial bioenergy is

163 needed. The fission processes are associated with the degradation of mitochondria. So fission is

164 induced when mitochondria are damaged. Deficiency of proteins involved in mitochondrial

165 fusion (Mfn2) reduces cellular respiration. Repression of Mfn2 is associated with a decrease in

166 cellular metabolism, and a deficiency of the proteins, which involved in mitochondrial fusion,

167 may indicate a decrease in cellular respiration and metabolism. Mitochondrial fusion may play a

168 role in the development of oxidative stress in metabolic syndrome. Mitochondrial fusion

169 mechanism rather than the fission mechanism was found is important for autophagy caused by

170 glucose starvation. The lack of fission due to suppression of the expression of Drp1 leads to a

171 loss of mtDNA and a decrease mitochondrial respiration in the cells. However, another study has

172 demonstrated that inhibition of Drp1 prevents a decrease in mitochondrial membrane potential

173 and the release of cytochrome $\mathrm{C}$ in cells. All these reports indicate that a shift to fission

174 processes is associated with mitochondrial dysfunction in the main tissues, such as skeletal

175 muscle and liver, involved in metabolic diseases associated with obesity [24]. Several studies

176 have shown that a change in the processes of mitochondrial fusion and fission can affect the

177 production of ROS in the mitochondria. The balance between mitochondrial fusion and fission

178 affects the important role in the regulation of mitochondrial energy and potentially contributes to

179 maintaining normoglycemia in obese patients.

180 Thus, the balance between fusion and fission of mitochondria plays a vital role in the regulation

181 of mitochondrial energy [25].

182

183

184

185

186

187

188

189

190

191

192

193

194

195

196

197

198

\section{Factors governing mtDNA copy number in insulin resistance}

Mitochondria respond to various metabolic disorders, such as oxidative stress, inflammation, glucotoxicity and lipotoxicity, biogenesis, and mitophagy, and the dynamics of mitochondria change (fusion and fission) [26]. Scientific evidence indicates the existence of a compensatory mechanism, which manifests as an increase in the number of copies of the mitochondrial genome under the influence of pro-inflammatory factors [27]. In particular, upon activation of TNF signalling pathway, the process of mitochondrial fission is triggered by DRP1 [27-29]. Studies in animal models of obesity have shown that with prolonged exposure to ROS, mitochondrial biogenesis changes. Perhaps that in the first months of life, the number of mtDNA copies of increases to compensate for oxidative stress. But over time, intracellular stocks are being depleted. In this regard, the number of mtDNA was significantly reduced [30].

A mutation of a gene in a single mtDNA molecule does not affect the nucleotide sequence of the same gene in any other mtDNA molecule [31]. It is assumed that the dysfunction of mtDNA is not associated with damage to single copies of mtDNA but is associated with changes in the copies of mtDNA in the cell $[32,33]$. In this regard, multiple copies of mtDNA may protect 
199

200

201

202

203

204

205

206

207

208

209

210

211

212

213

214

215

216

217

218

219

220

221

222

223

224

225

226

227

228

229

230

231

232

233

234

235

236

237

mitochondria from the severe damage to molecules under conditions of increased oxidative stress in the proximity of the ETC.

A mitochondrion is estimated to contain 2-10 mtDNA copies [34, 35], and the number of mitochondria in a cell varies depending on the needs of the tissue or organ. mtDNA has a higher mutation frequency than nuclear DNA [34, 35]. Normal mtDNA molecules and their mutated counterparts often display a 'co-existence' situation, termed heteroplasmy [34, 35]. Heteroplasmic patterns change in different tissues and are inherent to the original nature of the tissue. The percentage of heteroplasmy correlates with the penetrance of disease phenotypes. In our previous studies, it was shown that an increase in the number of mtDNA molecules in adipose tissue at different locations [mesentery (Mes), subcutaneous adipose tissue (SAT) and greater omentum (GO)] is associated with an increase in pro-inflammatory cytokines TNF- $\alpha$ Interleukin-6 (IL-6) and Interleukin-8 (IL-8) in the blood plasma of patients with obesity [36]. Other authors obtained data on a small but significant decrease in the number of mtDNA molecules corresponding with an increase in Body Mass Index (BMI) and age. In addition, the development of type 2 diabetes in patients with obesity did not depend on the number of mtDNA molecules [37]. However, Xu F. et al. (2012) showed that the amount of mtDNA negatively correlated with age, BMI, insulin level, homeostasis model assessment of insulin resistance (HOMA-IR) index, and cholesterol and triglyceride levels in patients with type 2 diabetes [38]. These conflicting data could be due to the small number and heterogeneity of the studied groups. However, they do confirm the involvement of mtDNA replication in the pathogenesis of obesity and type 2 diabetes.

We identified the dynamics of changes in mtDNA copy number with BMI. The number of mtDNA copy numbers in visceral adipose tissue (Mes and GO) increased in patients with BMI > $35 \mathrm{~kg} / \mathrm{m} 2$ relative to persons with a BMI of $30-34.9 \mathrm{~kg} / \mathrm{m} 2$ [39]. However, in all patients with a BMI $>40 \mathrm{~kg} / \mathrm{m} 2$, the number of mtDNA copies in the Mes was reduced compared with the number in the Mes of those with a BMI of 35-40.9 kg/m2 and was comparable to that of the control group. The decrease in mtDNA copy number found in our study could be considered a mechanism for adapting to the "soft disconnection" of the ETC, which also protects against the vicious cycle of ROS production. In addition, we identified higher levels of TNF- $\alpha$ in plasma and more significant mtDNA copy number in different tissues of patients with type 2 diabetes relative to levels and numbers in patients with obesity but not type 2 diabetes [39]. This result is logical because antioxidant protective enzymes in tissues of different locations function with different levels of efficacy [33], and tissues in people with obesity may exhibit different reactions to genotoxic agents than their counterparts without obesity. In mice, antioxidant protective enzymes in the pancreatic islets are expressed at a lower level than in other cell types [33]. In this regard, it is necessary to take into account the heterogeneity of responses by different tissues to cytotoxic damage. However, animal experiments have shown that changes in mtDNA copy number in peripheral blood leukocytes undergo processes similar to those in muscle tissue and hepatocytes, a finding that was also confirmed in our studies [39]. 
238 Perhaps the number of human of mtDNA molecules in human blood is an important indicator of

239

240

241

242

243

244

245

246

247

248

249

250

251

252

253

254

255

256

257

258

259

260

261

262

263

264

265

266

267

268

269

270

271

272

273

274

275

276

277

various metabolic disorders. Therefore, quantifying mtDNA in various biological samples can be used for predicting and evaluating the effectiveness of IR treatment.

\subsection{The role of pro-inflammatory factors-in the regulation of autophagy and mitochondrial fission and fusion}

Fusion and fission repair and regulate mitochondrial damage [40]. Fusion enriches damaged mitochondria with normal genome and proteins. This helps to avoid damage to the mitochondria. Fission, in turn, contributes to the destruction of damaged mitochondria by mitophagy [41]. The violation of mitochondrial quality control to remove damaged and dysfunctional mitochondria leads to the accumulation of damage associated with membrane patterns (DAMPs) released from injured cells, cell-free mtDNA, N-formyl peptides and Cardiolipin [42]. Mitochondrial DAMPs can bind and activate membrane or cytosolic pathogen recognition receptors (PRRs) such as nod like receptors (NLRs), toll-like receptors (TLR), like those recognized by pathogen-associated molecular pattern (PAMPs) [43]. Thus, it activates different early-phase inflammatory mediators like TNF- $\alpha$, interleukins, interferon-gamma (IFN- $\gamma$ ) and ROS/RNS [44]. In particular, TNF- $\alpha$, secreted by adipose tissue, hepatocytes and Kupffer cells, contributes significantly to mitochondrial dysfunction, contributing to the production of ROS and RNS by inducible nitric oxide synthase. Wang et al. (2012) reported that RIPK1/3 activates mitochondrial phosphoglycerate mutase/protein phosphatase - PGAM5, which dephosphorylates Drp1 at ser637 and promotes Drp1 fission activity $[45,46]$ - as a result, triggering TNF- $\alpha$ induced necrosis.

Thus, in turn, it will have a deleterious effect of damaging mitochondria. The net result is leading to the depletion of ATP production and promoting a switch to anaerobic glycolysis [47].

Therefore a combination of mitochondrial dysfunction plus upregulation of fission of mitochondria that produce ROS/RNS to an extent that exceeds antioxidant capacity, is lakely to be an initiating factors in inflammation, ageing, and age-related diseases [48].

In addition to fission and fusion, the process of mitophagy plays an important role in maintaining mitochondrial biogenesis. Mitophagy is a selective form of autophagy. Mitophagy helps to remove damaged or dysfunctional mitochondria through lysosomal degradation. Studies have demonstrated that loss of autophagy/mitophagy can lead to a build-up of cytosolic ROS and mtDNA. That, in turn, can activate immune signalling pathways that ultimately lead to the releases of inflammatory cytokines, including Interleukin- $1 \alpha$ (IL-1 $\alpha$ ), Interleukin-1 $\beta$ (IL-1 $\beta$ ), Interleukin-18 (IL-18), type I IFN and macrophage migration inhibitory factor (MIF) [49]. Moreover, the release of these cytokines can subsequently promote the release of others, including Interleukin-23 (IL-23) and Interleukin-17 (IL-17) [49].

An important process in maintaining normal mitochondrial function is the balance of automitophagy in the cell. For most differentiated tissues, proper control of the content, distribution, and activity of mitochondria is the key to maintaining normal cellular functioning. Because 
278 dysfunctional mitochondria contribute to the development of IR, autophagy is essential to 279 maintain the normal functioning of mitochondria and glucose metabolism. It has been shown 280 that, against the background of IR, skeletal muscle and liver cells suppress the autophagy of 281 organelles [50].

282 Mitochondria are central to apoptosis. Numerous studies have confirmed changes in the activity 283 of apoptotic factors in different tissues under normal and pathological conditions. Activation of 284 caspases, the main trigger of apoptosis, is controlled by Bcl-2 family proteins, which regulate the 285 release of caspase activators from mitochondria. There are two classes of proteins in the Bcl-2 286 family: anti-apoptotic proteins (Bcl-XL, Bcl-w, Mcl-1, A1, Bcl-Rambo, Bcl-L10, and Bcl-G) 287 and pro-apoptotic proteins (Bax, Bak, and Bok) [51;52;53].

288 It has established that these processes during oxidative stress are characterized by Bax activation 289 and the initiation of apoptosis [54]. Excessive ROS can also cause oxidative damage to mtDNA, 290 proteins, and phospholipids and induce apoptosis [53]. For these reasons, there will likely be 291 significant differences in cell type and the typical pathways that regulate the structure, function, 292 and content of mitochondria and the fate of cells exhibiting increased mitochondrial autophagy. 293 These factors complicate the study of mitophagy regulation, especially in pathological 294 conditions.

295

296

297

298

299

300

301

302

303

304

305

306

307

308

309

310

311

312

313

314

315

316

317

\section{Mitochondrial peptides}

The change in mtDNA copy number and the induction of fission and fusion depend on the energy needs of the cell. Mitochondria can serve as a matrix for the synthesis of mitochondrial peptides. It is known that 13 proteins, all components of the ETC, are encoded by mtDNA. However, recent studies have shown that mtDNA contains previously unknown short open reading frames (ORFs) that extend the genetic diversity of mitochondria [55]. As a rule, ORFencoded polypeptides of the nuclear genome possess significant biological activity, which can also be characteristic of mtDNA-encoded proteins.

The role of mitochondria as functional organelles and the signal molecules they produce is critical for cellular energy homeostasis, and mitochondrial dysfunction contributes to the pathogenesis of metabolic disorders. One of these molecules is protein humanin (Fig. 2) [56], which contributes to cell protection during oxidative stress by regulating mitochondrial functioning for more efficient ATP synthesis [56]. Increased mitochondrial biogenesis (in particular, the formation of new mitochondria, an increase in mtDNA copy number and the expression levels of mitochondrial transcription factors) in various insulin-dependent tissues may be one possible mechanism for optimizing bioenergy in cells under the action of humanin [56]. In contrast, humanin inhibits increases in mtDNA copy number in blood cells (Fig. 2) [14]. We previously showed that, in patients with a high BMI, an increase in circulating TNF- $\alpha$ contributes to the activation of a compensatory mechanism for maintaining mitochondrial biogenesis. This compensatory mechanism is associated with increased mtDNA copy number in the cells of subcutaneous adipose tissue and reduced mtDNA copy number in blood mononuclear 
318 cells [39]. In this regard, mitochondrial peptides play an important role in the functioning and 319 biogenesis of mitochondria.

320 A relatively recently identified peptide is MOTS-c (mitochondrial open reading frame of the $12 \mathrm{~S}$ 321 rRNA-c), which encodes a peptide of 16 amino acids. MOTS-c transcription occurs in the 322 cytoplasm, and the mechanism by which polyadenylated MOTS transcripts are exported out of 323 mitochondria is currently being investigated (Fig. 2) [55]. Although the functional activity of

324

325

326

327

328

329

330

331

332

333

334

335

336

337

338

339

340

341

342

343

344

345

346

347

348

349

350

351

352

353

354

355

356

357 MOTS-c has not been extensively studied, several studies have examined the mechanism of action of MOTS-c in cell cultures and animal models; however, to date, its properties have been extensively studied in humans. MOTS-c affects mitochondrial metabolism and insulin sensitivity [55], accelerates glucose uptake [55, 57], and activates insulin-dependent AKT kinase [14] in mouse skeletal muscle cells in culture. Also MOTS-c affects glucose production in the liver [14, $58]$.

One study showed that the level of MOTS-c in the circulation was reduced in male children with obesity and was associated with markers of IR and obesity [59]. Another research group measured plasma MOTS-c concentrations in healthy individuals and patients with obesity, and although they found that the protein level did not differ, they also found a negative correlation between plasma MOTS-c and the HOMA-IR index and the Matsuda index (alternative index of insulin sensitivity) [60]. However, the samples in these studies were limited to a small number of patients.

Under the influence of cellular stress, MOTS-c can translocate to the nucleus, where it affects the nuclear genome by changing its expression, which is an action particularly uncharacteristic of proteins encoded by mtDNA [61]. In the nucleus, MOTS-c binds to DNA and regulates gene transcription in combination with other transcription factors that contribute to protection against stress. This mitochondrial peptide is the first to have been discovered acting on transcriptional reactions in the nucleus in response to stress. MOTS-c regulates the expression of nuclear genes during metabolic stress in a 5'-adenosine monophosphate protein kinase (AMPK)-dependent manner (Fig. 2) [62]. Therefore, MOTS-c may have some characteristics of a "mitokine" factor (Fig. 2) [63, 64].

MOTS-c inhibits inflammatory pathways and improves the functioning of the endothelium [65]. Although the relationship between MOTS-c and the activation of the NF-kB receptor [65, 66] has been shown, the nature of this relationship has not yet been defined. MOTS-c inhibits the folate cycle involved in the regulation of carbohydrate metabolism by reducing the biosynthesis of purines, which leads to accumulation of the intermediate product of purine synthesis, 5amino-imidazole-4-carboxamide (AICAR), and activation of the metabolic regulator 5'-AMPactivated protein kinase (AMPK) (Fig. 2) [57]. Folate serves as a source of single carbon units for the methionine/homocysteine cycle by supplying 5-methyltetrahydrofolate used for the methylation of homocysteine such that it is reverted to methionine [67]. For example, decreased methionine can induce hepatic lipid accumulation by downregulating sterol regulatory elementbinding protein (Srebp1) mRNA and upregulating the expression of acetyl-CoA carboxylase 1 (Acc1) and fatty acid synthase (Fasn) mRNA, which are involved lipid synthesis in a hepatic cell 
358

359

360

361

362

363

364

365

366

367

368

369

370

371

372

373

374

375

376

377

378

379

380

381

382

383

384

385

386

387

388

389

390

391

392

393

394

395

396

397

[67]. The main risk factors for insulin resistance are hepatic steatosis. Moreover, insulin resistance can lead to the development of type 2 diabetes [67]. Thus, it was speculated that folate deficiency could induce glucose and lipid metabolism disorders.

It has been shown that MOTS-c works by regulating signalling pathways that act through AMPK and Sirtuin 1 (SIRT1). It should be noted that at high concentrations of MOTS-c, glucose is incorporated into the pentose phosphate pathway, which ensures the production of the substrate for the synthesis of purines, and is not oxidized through glycolysis [57]. With this in mind, the use of MOTS-c for the correction of carbohydrate metabolism is of great interest.

In addition, MOTS-c may serve as a potential therapeutic agent in the treatment of sepsis. Sepsis is characterized by uncontrolled inflammatory reactions to pathogenic bacterial infections, especially antibiotic-resistant strains. A study showed that MOTS-c increased survival rate and decreased bacterial load in infected mice, a decrease in the pro-inflammatory cytokines TNF- $\alpha$, IL-6, and IL-1 $\beta$ and an increase in the anti-inflammatory cytokine Interleukin-10 (IL-10) [68]. Mitochondria are directly involved in changes in carbohydrate metabolism and the development of IR. Given the promising positive effects of MOTS-c on the regulation of metabolic homeostasis, the therapeutic effects of this peptide in relation to obesity and diabetes are obvious. Future studies should focus on studying the metabolism and activity of MOTS-c in insulin-sensitive tissues in patients with type 2 diabetes mellitus to develop diagnostic methods and determine the therapeutic potential of this peptide.

\section{Therapeutic strategies based on mitochondrial regulation}

\subsection{Mitoproteomics in the treatment of insulin resistance}

The development of methods for proteomic analysis has allowed the investigation of changes to the composition of mitochondrial proteins, their redox state, and how the proteins interact with each other and regulate the functions and dynamics of mitochondria.

In mammalian cells, mtDNA encodes 13 proteins belonging to the mitochondrial respiratory chain, 2 ribosomal RNAs, and 22 transport RNAs, which determines the intramitochondrial translation code. This pathway is strictly regulated to maintain mitochondrial function [69]. The remaining mitochondrial proteins, which make up the other $99 \%$, are encoded by nuclear genes and depend on specific signals that direct them from the cytosol, where they are synthesized, to receptors on the surface of mitochondria and then to the corresponding mitochondrial compartment [70]. These include proteins involved in $\beta$-oxidation and protein transport; apoptotic factors; respiratory chain subunits, and the TCA components [71]. There are mechanisms by which mitochondrial proteins are imported into the organelle $[72,73$, 74]. The import of cytosolic-synthesized mitochondrial proteins usually requires cytosolic chaperones, such as heat-shock proteins (HSP) 70 and 90. These chaperones direct pre-proteins to the receptors of the translocase in the outer mitochondrial membrane (TOM) complex, mainly TOM70 and TOM20 [75]. 
398

399

400

401

402

403

404

405

406

407

408

409

410

411

412

413

414

415

416

417

418

419

420

421

422

423

424

425

426

427

428

429

430

431

432

433

434

435

436

Using a new approach, redox mitoproteomics, the authors proved that disruption of the mitochondrial complex assembly, together with the import of defective proteins, can be a potential cause of mitochondrial dysfunction in type 2 diabetes [76]; for example, changes in the pathways involved in the import of mitochondrial proteins at different levels, such as the TOM complex or the path of the mitochondrial intermembrane space assembly (MIA) [76, 77], may be involved. Also the authors established that complex IV (CIV) is a common target for the mitochondrial remodelling induced by ageing or type 2 diabetes [76].

The study of proteomic profiles provides unique information about thousands of mitochondrial proteins that can be used to assess mitochondrial functions that are related to the development of type 2 diabetes [78]. The large size of the proteome indicates that many metabolic and cellular pathways are active in mitochondria. Recent studies have shown significant tissue-specific differences in the composition of mitochondrial proteins [79, 80]. Approximately $1 / 3$ of all mitochondrial proteins are key components of the OXPHOS subunits and the TCA. Most subunits are tissue-specific.

In patients with obesity, studies of the mitoproteome within the fatty tissue of the greater omentum have shown that mitochondrial protein levels are reduced compared to those of the controls (without obesity). In addition, four proteins [citrate synthase, mitofilin, hydroxyacylCoA dehydrogenase/3-ketoacyl-CoA thiolase/enoyl-CoA hydratase (HADHA), and mitochondrial proton/calcium exchanger protein (LETM1)] were inversely correlated with BMI $[78,81]$. Chae et al. (2018) investigated the mitochondrial proteome of skeletal muscles in patients with type 2 diabetes [78] and compared the 1150 proteins discovered with the proteomes of mice and humans published elsewhere. It was established that 592 of the proteins had not yet been described, indicating that a wide range of mitoproteomic insulin-dependent tissues is involved in various pathophysiological processes.

A comparison of mitochondrial proteomes in patients with type 2 diabetes and those without diabetes revealed 335 differentially expressed proteins. Of these, 135 proteins were different in type 2 diabetes compared to those of the control (without type 2 diabetes), and 200 proteins were less active. Based on the results of extensive work in this area, the authors identified five potential proteins that indicate dysregulation of mitochondrial functions in type 2 diabetes: three proteins are associated with enhanced functions of the mitochondria-associated ER membrane (MAM), SORT, CALR, and RAB1A; and two proteins are associated with OXPHOS, mitochondrial NADH dehydrogenase ubiquinone iron-sulfur protein 3 (NDUFS3) and cyclooxygenase (COX2).

Thus, a comprehensive study of mitochondrial proteomes in insulin-sensitive tissues can provide a molecular basis for understanding the metabolic and cellular pathways that link mitochondrial dysregulation with type 2 diabetes.

\subsection{Therapy for regulating mitochondrial function}


437 Drugs and drug transport vehicles like aimed at mitochondria have significant potential because

438 of the presence of molecular target redundancy and their key role in metabolism [82].

439

440

441

442

443

444

445

446

447

448

449

450

451

452

453

454

455

456

457

458

459

460

461

462

463

464

465

466

467

468

469

470

471

472

473

474

475

Mitochondrial dysfunction can lead to pathologies that affect several pathways: ATP supply, mitochondrial biogenesis, mitochondrial fission and/or fusion, and it can affect the quality control of organelles. Drugs can directly affect the mitochondria or indirectly affect mitochondrial function by binding to regulatory targets in the cytosol or nucleus [83, 84]. Metformin is an oral hypoglycaemic biguanide drug currently used to treat type 2 diabetes. It inhibits mitochondrial complex I of the ETC and is an activator of AMP-activated protein kinase; therefore, metformin can reduce ATP production (Fig. 3) [85].

Low molecular weight mitochondrial uncouplers act as protonophores, transporting protons into the matrix independently of protein complexes or mediating molecular dissociation using proteins such as adenine nucleotide translocase (ANT) (Fig. 3) [59]. The use of low molecular weight uncouplers, such as dinitrophenol (DNP), which reduces the proton motive force $(\Delta p)$ in the inner mitochondrial membrane, makes OXPHOS less efficient, which helps burn excess fat tissue and reduces the mitochondrial ROS levels [86].

However, using pharmacological agents to uncoupled all mitochondria throughout the body may be a high-risk treatment. This is due to a might compromise energy homeostasis in tissues, especially the heart and brain. In humans treated orally and in animals, it was shown that the drug promoted a direct stimulation of cellular respiration and a consequent rise in body temperature, and also a significant decrease in body weight due to loss of body fat [87]. Therefore uncoupling can increase energy expenditure without compensatory mechanisms. Thus, the main shortcomings of the drug are a small difference between the effective and fatal doses of DNP, as well as a nonselective actions [86].

The therapeutic potential of mitochondrial uncouplers is associated with their dual role in increasing the oxidation of nutrients and decreasing the amount of ROS produced through the ETC. Although increased oxidation of nutrients contributes to weight loss and is a therapeutic strategy for the treatment of obesity and related metabolic diseases [88], mitochondrial ROS are associated with numerous pathologies, including ischaemia, reperfusion injury, inflammation, insulin resistance, neurodegenerative diseases, and many other pathologies. It is important to note that mitochondrial uncouplers prevent the production of ROS, which is beneficial, compared with antioxidants that remove ROS that has already been produced (Fig. 3). Therefore, a decrease in the production of mitochondrial ROS has significant therapeutic potential and advantages over antioxidant acceptors.

The balance of ROS and ATP depends on the rate of reduction of one electron $\mathrm{O} 2$ to a superoxide O2.- through the ETC and on the absorption rate through antioxidant systems. Mitochondria maximize energy production when ROS concentrations are high to create an intermediate redox state. Over time, the balance is lost, and ROS overflow occurs, which increases with distance from the optimal redox potential. At mitochondrial redox potentials rarely found, ROS production exceeds the absorption capacity such that, under oxidative 
476 conditions (for example, at high loads), the antioxidant protection is compromised and ultimately

477 eliminated [89].

478 The study of mitochondrial dynamics was proposed as a means to find the link between

479 mitochondrial dysfunction and IR [90]. Overexpressing proteins associated with fusion and

480 inhibiting proteins associated with fission strengthens the network of mitochondria and

481 suppresses IR, activating the IRS1-Akt pathway and inducing GLUT1/GLUT4 translocation to

482 the cell membrane. Pharmacological inhibition of DRP1 using the mitochondrial division

483 inhibitor (Mdivi-1) reduces IR and could initiate a new era in diabetes treatment [90]. Mdivi-1, a

484 chemical compound that weakens mitochondrial fission by selectively blocking the GTPase

485 activity of DRP1 [91], eliminates the dynamic imbalance of the mitochondrial genotype and

486 weakens insulin signalling in insulin-dependent tissues. A significant treatment Mdivi-1 restores

487 the balance of mitochondrial fusion and division in sepsis. The underlying molecular mechanism

488 may be that Mdivi-1 prevents the self-assembly of Drp1, which is required for mitochondrial

489 fission, and the selective inhibition of mitochondrial fission, in turn, suppresses mitochondrial

490 fusion defects $[92,93]$. Also, Mdivi-1 reduced the production of ROS and prevented the

491 occurrence of the endoplasmic reticulum stress in inflammation.

492 Mitochondrial biogenesis can be enhanced using drugs that indirectly alter the activity of PGC1 $\alpha$

493 [94, 95]. AMPK agonists, such as AICAR, activate PGC1 $\alpha$, mimicking enhanced mitochondrial

494 biogenesis [96]. Another approach is the use of the SIRT1 activators resveratrol and viniferin,

495 which activate PGC1 $\alpha$ by reversing acetylation [94]. A parallel approach to enhancing

496 mitochondrial biogenesis is to inhibit pathways that suppress it, such as that of the hypoxia-

497 induced factor $1 \alpha(\operatorname{HIF} 1 \alpha)[97,98]$.

498 The main problem in the development of drugs aimed at mitochondria is the need to create a

499 suitable way to deliver the desired molecule to its intended target. The development of drug

500 delivery systems that can permeate the mitochondrial double membrane is the key to the success

501 of mitochondrial therapy. Small molecules, such as ions, ATP, and proteins smaller than $5 \mathrm{kDa}$,

502 can freely diffuse through the channels of the outer membrane (proteins smaller than $10 \mathrm{kDa}$

503 through pass the TOM import gate); however, the mitochondrial inner membrane forms a barrier

504 that selectively transports molecules into the mitochondrial matrix [99].

505 There are delivery methods that use delocalized lipophilic cations. Such cations are connected to

506 the negatively charged mitochondrial matrix and can cross the membrane for accumulation in the

507 mitochondria [100]. Peptides containing the mitochondrial directional sequence, which

508 recognizes receptors on the mitochondrial membrane, have been used successfully to transport

509 chemical cargoes into the mitochondria [101]. Viruses are widely used in gene therapy as

510 carriers because of their innate ability to insert their genome into a host cell. For example, the

511 vector of adeno-associated viruses has been used to deliver genes to mitochondria [102];

512 however, despite numerous methods, few are sufficiently effective, and each has drawbacks.

513 In recent decades, the emergence of a completely new field of mitochondrial medicine is based

514 on an acknowledgement of the important role that mitochondria play in both human health and 
515 disease. Molecules for the development of these treatments must selectively act and accumulate 516 on the target site.

517 Therefore, it is necessary to investigate mitochondria as a target for treatment and to understand 518 the pathophysiology of the disease. Treatment strategies aimed at enhancing mitochondrial

519 function may represent important new approaches in the treatment of diabetes. The discovery of 520 the main mechanisms involved in the pathogenesis of diabetic complications provides a new 521 conceptual basis for future research. However, clinical trials will be required to show that the 522 compounds used in cell culture and animal studies apply to humans.

523

\section{Conclusions}

525 Mitochondria play an important role in the regulation of energy metabolism, ROS production, 526 apoptosis, and signal transduction. Mitochondria are dynamic organelles and can reprogram 527 themselves depending on the needs of the organism, regulating mtDNA stability, respiratory

528

529

530

531

532

533

534

535

536

537

538

539

540

\section{Acknowledgements}

542 No acknowledgments

543

\section{Funding}

545 The research was supported by Russian Foundation for Basic Research [No. 18-015-00084-a to 546 Larisa Litvinova, by Russian Foundation for Basic Research and Kaliningrad Region [No.19-

547 415-393004 - r_mol_a to Daria Skuratovskaia, No.19-44-390005 - r_a to Daria Skuratovskaia]; 548 state assignment [No. 075-03-2020-080 to Larisa Litvinova]; state support of leading scientific 549 schools of the Russian Federation [No. 2495.2020.7 to Larisa Litvinova].

\section{References}


552 1. Jørgensen W, Rud KA, Mortensen OH, Frandsen L, Grunnet N, Quistorff B. Your

553 mitochondria are what you eat: a high-fat or a high-sucrose diet eliminates metabolic flexibility

554 in isolated mitochondria from rat skeletal muscle. Physiol Rep. 2017;5.

555 2. Stanley WC, Recchia FA, Lopaschuk GD. Myocardial substrate metabolism in the normal and

556 failing heart. Physiol Rev. 2005;85:1093-129.

557 3. Guo R, Gu J, Zong S, Wu M, Yang M. Structure and mechanism of mitochondrial electron

558 transport chain. Biomed J. 2018;41:9-20.

559 4. Lee JH, Park A, Oh KJ, Lee SC, Kim WK, Bae KH. The Role of Adipose Tissue

560 Mitochondria: Regulation of Mitochondrial Function for the Treatment of Metabolic Diseases.

561 Int J Mol Sci. 2019;20(19):4924.

562 5. Snodgrass RG, Boß M, Zezina E, Weigert A, Dehne N, Fleming I, Brüne B, \& Namgaladze,

563 D. Hypoxia Potentiates Palmitate-induced Pro-inflammatory Activation of Primary Human

564 Macrophages. J Biol Chem. 2016;291:413-24.

565 6. Bergmann K, Sypniewska G. Diabetes as a complication of adipose tissue dysfunction. Is

566 there a role for potential new biomarkers? Clin Chem Lab Med. 2013;51:177-85.

567 7. Litvinova L, Atochin DN, Fattakhov N, Vasilenko M, Zatolokin P, Kirienkova E. Nitric oxide

568 and mitochondria in metabolic syndrome. Front Physiol. 2015;6:20.

569 8. Münzel T, Gori T, Bruno RM, Taddei S. Is oxidative stress a therapeutic target in

570 cardiovascular disease? Eur Heart J. 2010;31:2741-8.

571 9. Pagel-Langenickel I, Bao J, Pang L, Sack MN. The role of mitochondria in the

572 pathophysiology of skeletal muscle insulin resistance. Endocr Rev. 2010;31:25-51.

573 10. Yuzefovych LV, Musiyenko SI, Wilson GL, Rachek LI. Mitochondrial DNA damage and

574 dysfunction, and oxidative stress are associated with endoplasmic reticulum stress, protein

575 degradation and apoptosis in high fat diet-induced insulin resistance mice. PloS One.

576 2013;8:e54059.

577 11. Indo HP, Yen H-C, Nakanishi I, Matsumoto K, Tamura M, Nagano Y, Matsui H, Gusev O,

578 Cornette R, Okuda T, Minamiyama Y, Ichikawa H, Suenaga S, Oki M, Sato T, Ozawa T, Clair

579 DK, \& Majima H J. A mitochondrial superoxide theory for oxidative stress diseases and aging. J

580 Clin Biochem Nutr. 2015;56:1-7.

581 12. Mishra M, Lillvis J, Seyoum B, Kowluru RA. Peripheral Blood Mitochondrial DNA Damage

582 as a Potential Noninvasive Biomarker of Diabetic Retinopathy. Invest Ophthalmol Vis Sci.

583 2016;57:4035-44.

584 13. Herbst A, Wanagat J, Cheema N, Widjaja K, McKenzie D, Aiken JM. Latent mitochondrial

585 DNA deletion mutations drive muscle fiber loss at old age. Aging Cell. 2016;15:1132-9.

586 14. Kim S-J, Xiao J, Wan J, Cohen P, Yen K. Mitochondrially derived peptides as novel

587 regulators of metabolism. J Physiol. 2017;595:6613-21.

588 15. Jiang W, Li R, Zhang Y, Wang P, Wu T, Lin J, Yu J, \& Gu M. Mitochondrial DNA

589 Mutations Associated with Type 2 Diabetes Mellitus in Chinese Uyghur Population. Sci Rep.

590 2017;7:16989.

591 16. Ye J. Mechanisms of insulin resistance in obesity. Front Med. 2013;7:14-24. 
592 17. Serra D, Mera P, Malandrino MI, Mir JF, Herrero L. Mitochondrial fatty acid oxidation in 593 obesity. Antioxid Redox Signal. 2013;19:269-84.

594 18. Maechler P, Wollheim CB. Mitochondrial function in normal and diabetic beta-cells. Nature. 595 2001;414:807-12.

596 19. Youle RJ, van der Bliek AM. Mitochondrial fission, fusion, and stress. Science.

597 2012;337:1062-5.

598 20. Losón OC, Song Z, Chen H, Chan DC. Fis1, Mff, MiD49, and MiD51 mediate Drp1

599 recruitment in mitochondrial fission. Mol Biol Cell. 2013;24:659-67.

600 21. Nan J, Hu H, Sun Y, Zhu L, Wang Y, Zhong Z, Zhao J, Zhang N, Wang Y, Wang Y, Ye J,

601 Zhang L, Hu X, Zhu W, \& Wang J. TNFR2 Stimulation Promotes Mitochondrial Fusion via

602 Stat3- and NF-kB-Dependent Activation of OPA1 Expression. Circ Res. 2017;121:392-410.

603 22. Pohjoismäki JL, Wanrooij S, Hyvärinen AK, Goffart S, Holt IJ., Spelbrink JN, Jacobs HT.

604 Alterations to the expression level of mitochondrial transcription factor A, TFAM, modify the

605 mode of mitochondrial DNA replication in cultured human cells. Nucleic acids research. 2006;

606 34: 5815-5828.

607 23. Gerald W. Dorn II. Evolving Concepts of Mitochondrial Dynamics. Annual Review of

608 Physiology. 2019;81:1-17.

609 24. Wu C, Yao W, Kai W, Liu W, Wang W, Li S, Chen Y, Wu X, Wang L, Li Y, Tong J, Qian J,

610 Zhang L, Hong Z, \& Yi C. Mitochondrial Fusion Machinery Specifically Involved in Energy

611 Deprivation-Induced Autophagy. Front Cell Dev Biol. 2020;7;8:221.

612 25. Hu Y, Chen H, Zhang L, Lin X, Li X, Zhuang H, Fan H, Meng T, He Z, Huang H, Gong Q,

613 Zhu D, Xu Y, He P, Li L, \& Feng D. The AMPK-MFN2 axis regulates MAM dynamics and

614 autophagy induced by energy stresses. Autophagy. 2020;19:1-15.

615 26. Koliaki C, Roden M. Alterations of Mitochondrial Function and Insulin Sensitivity in Human

616 Obesity and Diabetes Mellitus. Annu Rev Nutr. 2016;36:337-67.

617 27. Xie Q, Deng Y, Huang C, Liu P, Yang Y, Shen W \& Gao P. Chemerin-induced

618 mitochondrial dysfunction in skeletal muscle. J Cell Mol Med. 2015;19:986-95.

619 28. Zhang B, Davidson MM, Zhou H, Wang C, Walker WF, Hei TK. Cytoplasmic irradiation

620 results in mitochondrial dysfunction and DRP1-dependent mitochondrial fission. Cancer Res.

$621 \quad 2013 ; 73: 6700-10$.

622 29. KEGG PATHWAY: TNF signaling pathway - Homo sapiens (human).

623 https://www.genome.jp/kegg-bin/show_pathway?hsa04668. Accessed 1 Aug 2019.

624 30. Wang P-W, Kuo H-M, Huang H-T, Chang AYW, Weng S-W, Tai M-H, Chuang J H, Chen I

625 Y, Huang SC, Lin TK \& Liou CW. Biphasic response of mitochondrial biogenesis to oxidative

626 stress in visceral fat of diet-induced obesity mice. Antioxid Redox Signal. 2014;20:2572-88.

627 31. Sivitz WI, Yorek MA. Mitochondrial dysfunction in diabetes: from molecular mechanisms to

628 functional significance and therapeutic opportunities. Antioxid Redox Signal. 2010;12:537-77.

629 32. Abdullaev SA, Antipova VN, Gaziev AI. Extracellular mutant mitochondrial DNA content is

630 dramatically elevated in the blood plasma of irradiated mice. Mol Biol. 2009;43:990. 
631 33. Marín-García J. Mitochondrial DNA repair: a novel therapeutic target for heart failure. Heart 632 Fail Rev. 2016;21:475-87.

633 34. Duan M, Tu J, Lu Z. Recent Advances in Detecting Mitochondrial DNA Heteroplasmic 634 Variations. Mol J Synth Chem Nat Prod Chem. 2018;23. doi:10.3390/molecules23020323.

635 35. Avital G, Buchshtav M, Zhidkov I, Tuval (Feder) J, Dadon S, Rubin E, Glass D, Spector TD, 636 Mishmar D. Mitochondrial DNA heteroplasmy in diabetes and normal adults: role of acquired 637 and inherited mutational patterns in twins. Hum Mol Genet. 2012;21:4214-24.

638 36. Litvinova L, Zatolokin P, Vulf M, Mazunin I, Skuratovskaia D. The relationship between the 639 mtDNA copy number in insulin-dependent tissues and markers of endothelial dysfunction and 640 inflammation in obese patients. BMC Med Genomics. 2019;12 Suppl 2:41.

641 37. Kaaman M, Sparks LM, van Harmelen V, Smith SR, Sjölin E, Dahlman I, Arner P. Strong 642 association between mitochondrial DNA copy number and lipogenesis in human white adipose 643 tissue. Diabetologia. 2007;50:2526-33.

644 38. Xu FX, Zhou X, Shen F, Pang R, Liu SM. Decreased peripheral blood mitochondrial DNA 645 content is related to $\mathrm{HbA1c}$, fasting plasma glucose level and age of onset in type 2 diabetes 646 mellitus. Diabet Med J Br Diabet Assoc. 2012;29:e47-54.

647 39. Skuratovskaia D, Zatolokin P, Vulf M, Mazunin I, Litvinova L. Interrelation of chemerin and 648 TNF- $\alpha$ with mtDNA copy number in adipose tissues and blood cells in obese patients with and 649 without type 2 diabetes. BMC Med Genomics. 2019;12 Suppl 2:40.

650 40. Geto Z, Molla MD, Challa F, Belay Y, Getahun T. Mitochondrial Dynamic Dysfunction as a

651 Main Triggering Factor for Inflammation Associated Chronic Non-Communicable Diseases. J

652 Inflamm Res. 2020;13:97-107.

653 41. Westermann B. Mitochondrial fusion and fission in cell life and death. Nat Rev Mol Cell 654 Biol . 2010;11:872-884.

655 42. Zhang Q, Raoof M, Chen Y, Sumi Y, Sursal T, Junger W, Brohi K, Itagaki K, Hauser CJ. 656 Circulating mitochondrial DAMPs cause inflammatory responses to injury. Nature.

657 2010;464(7285):104-107.

658 43. Collins LV, Hajizadeh S, Holme E, Jonsson IM, Tarkowski A. Endogenously oxidized 659 mitochondrial DNA induces in vivo and in vitro inflammatory responses. J Leukoc Biol.

660 2004;75(6):995-1000.

661 44. Picca A, Lezza AMS, Leeuwenburgh C, Pesce V, Calvani R, Landi F, Bernabei R, Marzetti

662 E. Fueling Inflamm-Aging through Mitochondrial Dysfunction: Mechanisms and Molecular 663 Targets. Int J Mol Sci. 2017;18(5):933.

664 45. Wang Z, Jiang H, Chen S, Du F, Wang X. The mitochondrial phosphatase PGAM5 functions 665 at the convergence point of multiple necrotic death pathways. Cell. 2012;148(1-2):228-243.

666 46. Luo F, Herrup K, Qi X, Yang Y. Inhibition of Drp1 hyper-activation is protective in animal 667 models of experimental multiple sclerosis. Exp Neurol. 2017;292:21-34.

668 47. Morris G, Berk M. The many roads to mitochondrial dysfunction in neuroimmune and 669 neuropsychiatric disorders. BMC Med. 2015;13:68. 
670 48. Hernández-Aguilera A, Rull A, Rodríguez-Gallego E, Luciano-Mateo F, Camps J.

671 Mitochondrial dysfunction: a basic mechanism in inflammation-related non-communicable

672 diseases and therapeutic opportunities. Mediators Inflamm. 2013;2013:135698.

673 49. Harris J, Deen N, Zamani S, Hasnat MA. Mitophagy and the release of inflammatory

674 cytokines. Mitochondrion. 2018; 41: 2-8.

675 50. Cho DK, Choi DH, Cho JY. Effect of treadmill exercise on skeletal muscle autophagy in rats 676 with obesity induced by a high-fat diet. J Exerc Nutr Biochem. 2017;21:26-34.

677 51. Estaquier J, Vallette F, Vayssiere J-L, Mignotte B. The mitochondrial pathways of apoptosis. 678 Adv Exp Med Biol. 2012;942:157-83.

679 52. Parrish AB, Freel CD, Kornbluth S. Cellular mechanisms controlling caspase activation and 680 function. Cold Spring Harb Perspect Biol. 2013;5.

681 53. Cao L, Quan X-B, Zeng W-J, Yang X-O, Wang M-J. Mechanism of Hepatocyte Apoptosis. J 682 Cell Death. 2016;9:19-29.

683 54. Petrasek J, Iracheta-Vellve A, Csak T, Satishchandran A, Kodys K, Kurt-Jones EA, 684 Fitzgerald KA, Szabo G. STING-IRF3 pathway links endoplasmic reticulum stress with 685 hepatocyte apoptosis in early alcoholic liver disease. Proc Natl Acad Sci U S A.

686 2013;110:16544-9.

687 55. Lee C, Kim KH, Cohen P. MOTS-c: A novel mitochondrial-derived peptide regulating

688 muscle and fat metabolism. Free Radic Biol Med. 2016;100:182-7.

689 56. Sreekumar PG, Ishikawa K, Spee C, Mehta HH, Wan J, Yen K, Cohen P, Kannan R, Hinton

690 DR. The Mitochondrial-Derived Peptide Humanin Protects RPE Cells From Oxidative Stress,

691 Senescence, and Mitochondrial Dysfunction. Invest Ophthalmol Vis Sci. 2016;57:1238-53.

692 57. Lee C, Zeng J, Drew BG, Sallam T, Martin-Montalvo A, Wan J, Kim SJ, Mehta H, Hevener

693 AL, de Cabo R, Cohen P. The mitochondrial-derived peptide MOTS-c promotes metabolic

694 homeostasis and reduces obesity and insulin resistance. Cell Metab. 2015;21:443-54.

695 58. Li H-X, Chen K-L, Wang H-Y, Tang C-B, Xu X-L, Zhou G-H. Chemerin inhibition of

696 myogenesis and induction of adipogenesis in $\mathrm{C} 2 \mathrm{C} 12$ myoblasts. Mol Cell Endocrinol.

$697 \quad 2015 ; 414: 216-23$.

698 59. Childress ES, Alexopoulos SJ, Hoehn KL, Santos WL. Small Molecule Mitochondrial

699 Uncouplers and Their Therapeutic Potential. J Med Chem. 2018;61:4641-55.

700 60. Cataldo LR, Fernández-Verdejo R, Santos JL, Galgani JE. Plasma MOTS-c levels are

701 associated with insulin sensitivity in lean but not in obese individuals. J Investig Med Off Publ

702 Am Fed Clin Res. 2018;66:1019-22.

703 61. Mangalhara KC, Shadel GS. A Mitochondrial-Derived Peptide Exercises the Nuclear Option.

704 Cell Metab. 2018;28:330-1.

705 62. Kim KH, Son JM, Benayoun BA, Lee C. The Mitochondrial-Encoded Peptide MOTS-c

706 Translocates to the Nucleus to Regulate Nuclear Gene Expression in Response to Metabolic

707 Stress. Cell Metab. 2018;28:516-524.e7.

708 63. Yong CQY, Tang BL. A Mitochondrial Encoded Messenger at the Nucleus. Cells. 2018;7.

709 doi:10.3390/cells7080105. 
710 64. Mendelsohn AR, Larrick JW. Mitochondrial-Derived Peptides Exacerbate Senescence.

711 Rejuvenation Res. 2018;21:369-73.

712 65. Li H, Ren K, Jiang T, Zhao G-J. MOTS-c attenuates endothelial dysfunction via suppressing 713 the MAPK/NF-kB pathway. Int J Cardiol. 2018;268:40.

714 66. Ming W, Lu G, Xin S, Huanyu L, Yinghao J, Xiaoying L, Chengming X, Banjun R, Li W,

715 Zifan L. Mitochondria related peptide MOTS-c suppresses ovariectomy-induced bone loss via

716 AMPK activation. Biochem Biophys Res Commun. 2016;476:412-9.

717 67. Zhao M, Yuan MM, Yuan L, Huang LL, Liao JH, Yu XL, Su C, Chen YH, Yang YY, Yu H,

$718 \mathrm{Xu} \mathrm{X}$. Chronic folate deficiency induces glucose and lipid metabolism disorders and subsequent

719 cognitive dysfunction in mice. PLoS ONE. 2018;13. doi:10.1371/journal.pone.0202910.

720 68. Zhai D, Ye Z, Jiang Y, Xu C, Ruan B, Yang Y, Lei X, Xiang A, Lu H, Zhu Z, Yan Z, Wei D,

721 Li Q, Wang L, Lu Z. MOTS-c peptide increases survival and decreases bacterial load in mice

722 infected with MRSA. Mol Immunol. 2017;92:151-60.

723 69. Gustafsson CM, Falkenberg M, Larsson N-G. Maintenance and Expression of Mammalian

724 Mitochondrial DNA. Annu Rev Biochem. 2016;85:133-60.

725 70. Pfanner N, Warscheid B, Wiedemann N. Mitochondrial proteins: from biogenesis to

726 functional networks. Nat Rev Mol Cell Biol. 2019;20:267-84.

727 71. Gómez-Serrano M, Camafeita E, Loureiro M, Peral B. Mitoproteomics: Tackling

728 Mitochondrial Dysfunction in Human Disease. Oxid Med Cell Longev. 2018;2018:1435934.

729 72. Chinnery PF, Hudson G. Mitochondrial genetics. Br Med Bull. 2013;106:135-59.

730 73. Chacinska A, Koehler CM, Milenkovic D, Lithgow T, Pfanner N. Importing mitochondrial

731 proteins: machineries and mechanisms. Cell. 2009;138:628-44.

732 74. Stojanovski D, Bragoszewski P, Chacinska A. The MIA pathway: a tight bond between

733 protein transport and oxidative folding in mitochondria. Biochim Biophys Acta.

734 2012;1823:1142-50.

735 75. Neupert W, Herrmann JM. Translocation of proteins into mitochondria. Annu Rev Biochem.

$736 \quad 2007 ; 76: 723-49$.

737 76. Gómez-Serrano M, Camafeita E, López JA, Rubio MA, Bretón I, García-Consuegra I, Lago

738 J, Sánchez-Pernaute A, Torres A, Vázquez J, Peral B. Differential proteomic and oxidative

739 profiles unveil dysfunctional protein import to adipocyte mitochondria in obesity-associated

740 aging and diabetes. Redox Biol. 2017;11:415-28.

741 77. Stojanovski D, Müller JM, Milenkovic D, Guiard B, Pfanner N, Chacinska A. The MIA

742 system for protein import into the mitochondrial intermembrane space. Biochim Biophys Acta.

$743 \quad 2008 ; 1783: 610-7$.

744 78. Chae S, Kim S-J, Do Koo Y, Lee JH, Kim H, Ahn BY, Ha YC, Kim YH, Jang MG, Koo KH,

745 Choi SH, Lim S, Park YJ, Jang HC, Hwang D, Lee SW, Park KS. A mitochondrial proteome

746 profile indicative of type 2 diabetes mellitus in skeletal muscles. Exp Mol Med. 2018;50:129.

747 79. Mootha VK, Bunkenborg J, Olsen JV, Hjerrild M, Wisniewski JR, Stahl E, Bolouri MS, Ray

748 HN, Sihag S, Kamal M, Patterson N, Lander ES, Mann M. Integrated analysis of protein 
749 composition, tissue diversity, and gene regulation in mouse mitochondria. Cell. 2003;115:629-

75040.

751 80. Pagliarini DJ, Calvo SE, Chang B, Sheth SA, Vafai SB, Ong S-E, Walford GA, Sugiana C,

752 Boneh A, Chen WK, Hill DE, Vidal M, Evans JG, Thorburn DR, Carr SA, Mootha VK. A

753 mitochondrial protein compendium elucidates complex I disease biology. Cell. 2008;134:112-

75423.

755 81. Lindinger PW, Christe M, Eberle AN, Kern B, Peterli R, Peters T, Jayawardene KJ, Fearnley

756 IM, Walker JE. Important mitochondrial proteins in human omental adipose tissue show reduced 757 expression in obesity. J Proteomics. 2015;124:79-87.

758 82. Murphy MP, Hartley RC. Mitochondria as a therapeutic target for common pathologies. Nat 759 Rev Drug Discov. 2018;17:865-86.

760 83. Nightingale H, Pfeffer G, Bargiela D, Horvath R, Chinnery PF. Emerging therapies for 761 mitochondrial disorders. Brain J Neurol. 2016;139 Pt 6:1633-48.

762 84. Smith RAJ, Hartley RC, Cochemé HM, Murphy MP. Mitochondrial pharmacology. Trends 763 Pharmacol Sci. 2012;33:341-52.

764 85. Hawley SA, Gadalla AE, Olsen GS, Hardie DG. The antidiabetic drug metformin activates 765 the AMP-activated protein kinase cascade via an adenine nucleotide-independent mechanism.

766 Diabetes. 2002;51:2420-5.

767 86. Harper JA, Dickinson K, Brand MD. Mitochondrial uncoupling as a target for drug 768 development for the treatment of obesity. Obes Rev Off J Int Assoc Study Obes. 2001;2:255-65.

769 87. Magne H, Mayer A, Plantefol L. Studies on the action of dinitrophenol 1-2-4 (Thermol).

770 Ann Physiol Physicochem Biol 1932; 8: 1-167.

771 88. Perry RJ, Kim T, Zhang X-M, Lee H-Y, Pesta D, Popov VB, Zhang D, Rahimi Y, Jurczak

772 MJ, Cline GW, Spiegel DA, Shulman GI. Reversal of hypertriglyceridemia, fatty liver disease,

773 and insulin resistance by a liver-targeted mitochondrial uncoupler. Cell Metab. 2013;18:740-8.

774 89. Aon MA, Cortassa S, O’Rourke B. Redox-optimized ROS balance: a unifying hypothesis.

775 Biochim Biophys Acta. 2010;1797:865-77.

776 90. Lin H-Y, Weng S-W, Chang Y-H, Su Y-J, Chang C-M, Tsai C-J, Shen F-C, Chuang J-H, Lin

777 T-K, Liou C-W, Lin C-Y, Wang P-W. The Causal Role of Mitochondrial Dynamics in

778 Regulating Insulin Resistance in Diabetes: Link through Mitochondrial Reactive Oxygen

779 Species. Oxid Med Cell Longev. 2018;2018:7514383.

780 91. Cassidy-Stone A, Chipuk JE, Ingerman E, Song C, Yoo C, Kuwana T, Kurth MJ, Shaw JT, 781 Hinshaw JE, Green DR, Nunnari J. Chemical inhibition of the mitochondrial division dynamin 782 reveals its role in Bax/Bak-dependent mitochondrial outer membrane permeabilization. Dev Cell. 783 2008;14:193-204.

784 92. Tanaka A., Youle R. J. A chemical inhibitor of DRP1 uncouples mitochondrial fission and 785 apoptosis. Molecular Cell. 2008;29(4):409-410.

786 93. Pagliuso A., Cossart P., Stavru F. The ever-growing complexity of the mitochondrial fission 787 machinery. Cellular and Molecular Life Sciences. 2018;75(3):355-374. 
788 94. Whitaker RM, Corum D, Beeson CC, Schnellmann RG. Mitochondrial Biogenesis as a

789 Pharmacological Target: A New Approach to Acute and Chronic Diseases. Annu Rev Pharmacol

790 Toxicol. 2016;56:229-49.

791 95. Scarpulla RC. Metabolic control of mitochondrial biogenesis through the PGC-1 family

792 regulatory network. Biochim Biophys Acta. 2011;1813:1269-78.

793 96. Viscomi C, Bottani E, Civiletto G, Cerutti R, Moggio M, Fagiolari G, Schon EA, Lamperti

$794 \mathrm{C}$, Zeviani M. In vivo correction of COX deficiency by activation of the AMPK/PGC-1 $\alpha$ axis.

795 Cell Metab. 2011;14:80-90.

796 97. Zhang H, Gao P, Fukuda R, Kumar G, Krishnamachary B, Zeller KI, Dang CV, Semenza

797 GL. HIF-1 inhibits mitochondrial biogenesis and cellular respiration in VHL-deficient renal cell

798 carcinoma by repression of C-MYC activity. Cancer Cell. 2007;11:407-20.

799 98. Semenza GL. Oxygen-dependent regulation of mitochondrial respiration by hypoxia-

800 inducible factor 1. Biochem J. 2007;405:1-9.

801 99. Jang Y-H, Lim K-I. Recent Advances in Mitochondria-Targeted Gene Delivery. Mol Basel

802 Switz. 2018;23.

803 100. Murphy MP. Selective targeting of bioactive compounds to mitochondria. Trends

804 Biotechnol. 1997; 15:326-30.

805 101. Vestweber D, Schatz G. DNA-protein conjugates can enter mitochondria via the protein

806 import pathway. Nature. 1989; 338: 170-2.

807 102. Yu H, Ozdemir SS, Koilkonda RD, Chou T-H, Porciatti V, Chiodo V, Boye SL, Hauswirth

808 WW, Lewin AS, Guy J. Mutant NADH dehydrogenase subunit 4 gene delivery to mitochondria 809 by targeting sequence-modified adeno-associated virus induces visual loss and optic atrophy in 810 mice. Mol Vis. 2012;18:1668-83.

811 


\section{Figure 1}

Figure 1. The development of mitochondrial dysfunction and insulin resistance on the background of obesity.

A - Adipose tissue secretes pro-inflammatory mediators and free fatty acids (FFAs) due to hypoxia, generating chronic inflammation. High levels of FFAs, DAMPS/PAMPS, leptin, TNF $\alpha$, and IL-6 block insulin signalling. B - In mitochondria, the electron transport chain (ETC) is disrupted, which is characterized by a decrease in ATP production and an increase in ROS. Oxidative stress damages DNA, RNA, proteins, and, in particular, mtDNA, which leads to the formation of deletions and a decrease in the functional activity of mitochondria. Under the action of oxidative stress, the process of mitochondrial fission and fusion is launched. These processes mix the contents of partially damaged mitochondria and contribute to quality control by enabling the removal of damaged mitochondria and creating new mitochondria. Impaired mitochondrial function in insulin-dependent tissues, in addition to contributing to the formation of inflammation, leads to an energy crisis that underlies insulin resistance. This figure has been created by modifying the templates from Servier Medical Art (Servier Medical Art, 2020); CC BY 3.0 Unported. 


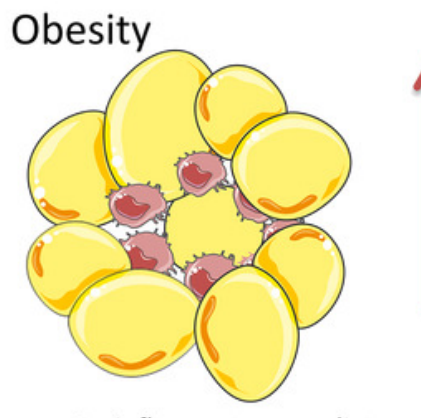

Proinflammatory mediators Chemokines

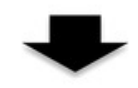

\section{Insulin Resistance}
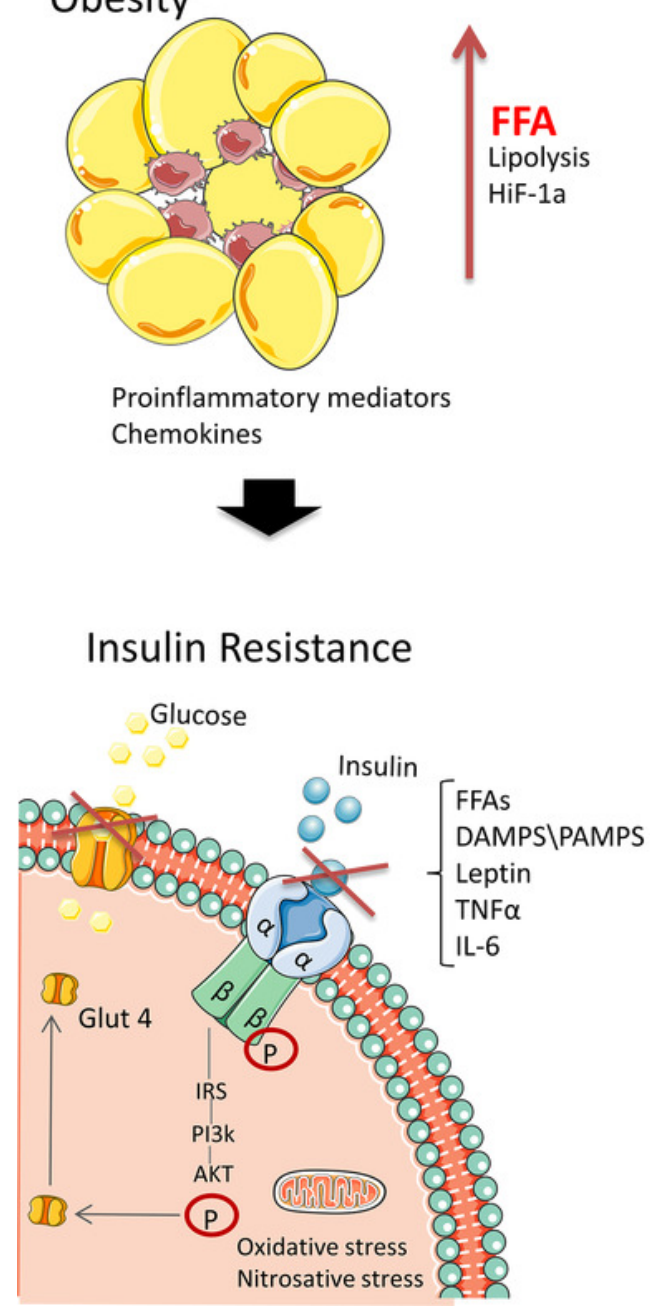

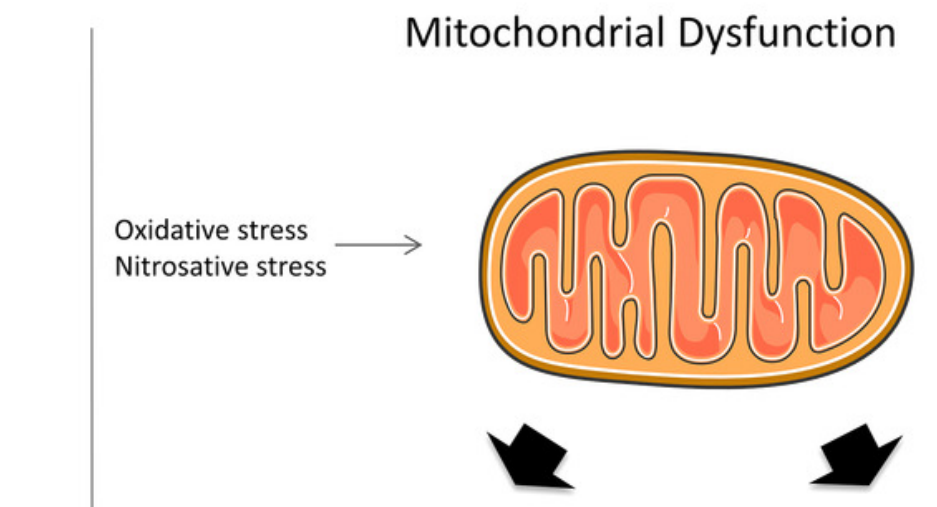

Dysfunction electronic transport chain

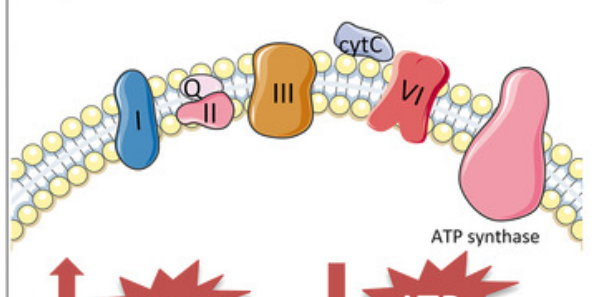

Mitochondrial biogenesis
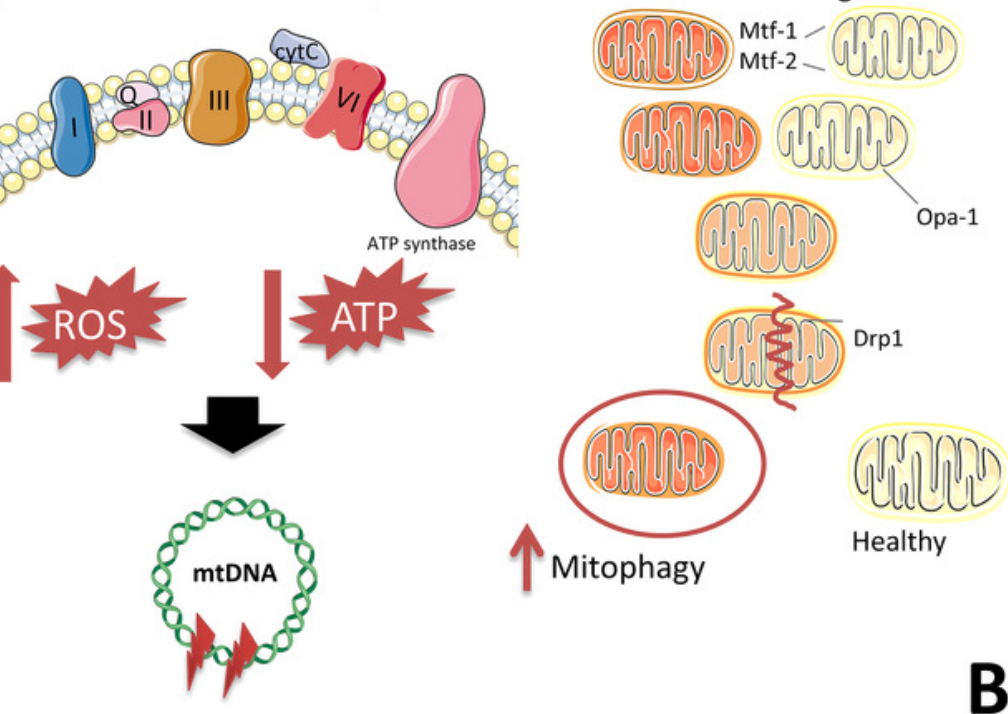


\section{Figure 2}

Figure 2. Diagram of the effects of mitochondrial peptides MOTS-c and humanin.

The peptide has positive effects in the regulation of carbohydrate metabolism and can interact with the nuclear genome. nDNA - nuclear DNA, mtDNA - mitochondrial DNA. This figure has been created by modifying the templates from Servier Medical Art (Servier Medical Art, 2020); CC BY 3.0 Unported. 


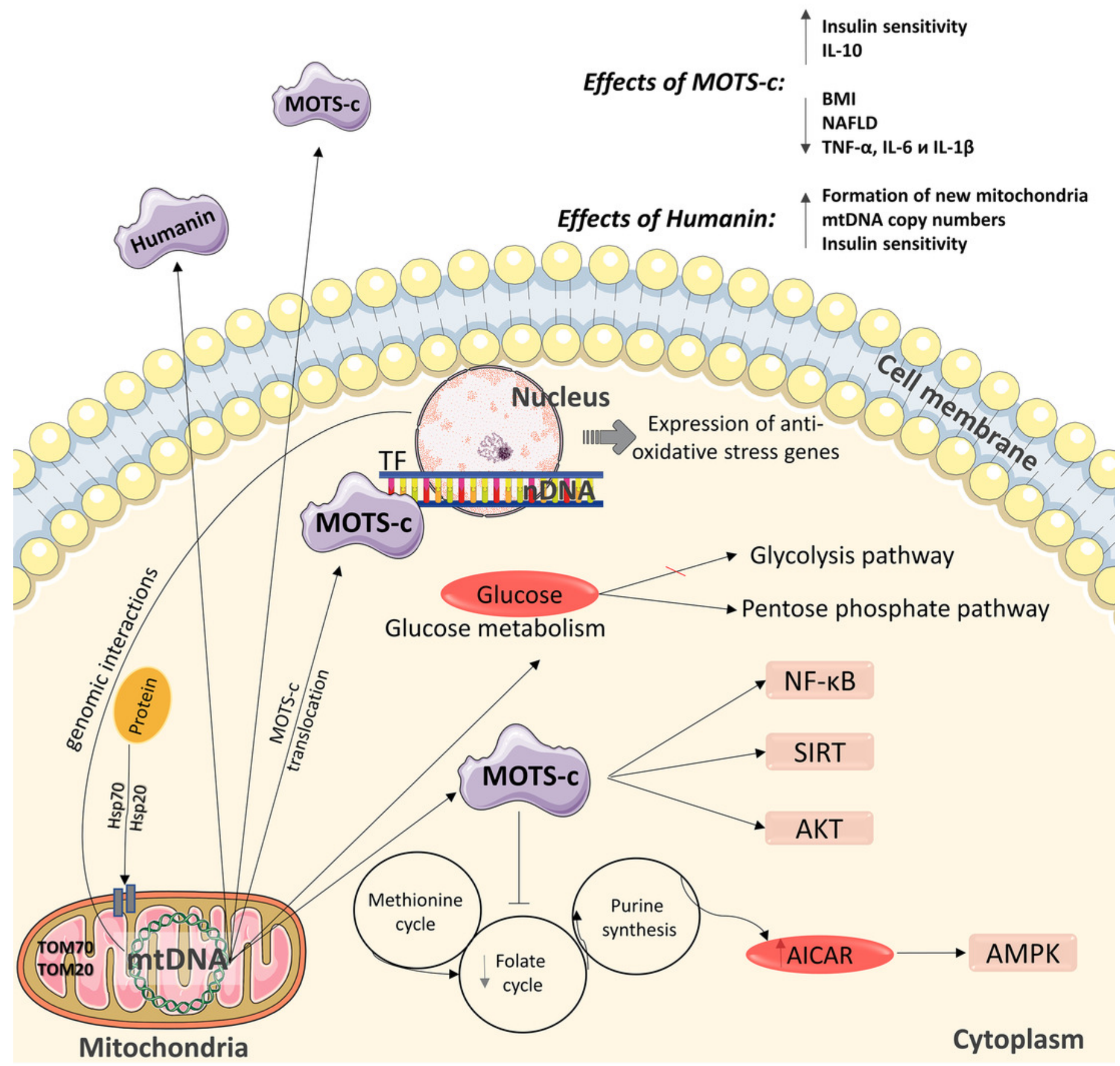


Figure 3

Figure 3. Target drags for the regulation of biogenesis and functioning of mitochondria.

The effects of drugs regulating mitochondrial function. This figure has been created by modifying the templates from Servier Medical Art (Servier Medical Art, 2020); CC BY 3.0 Unported.

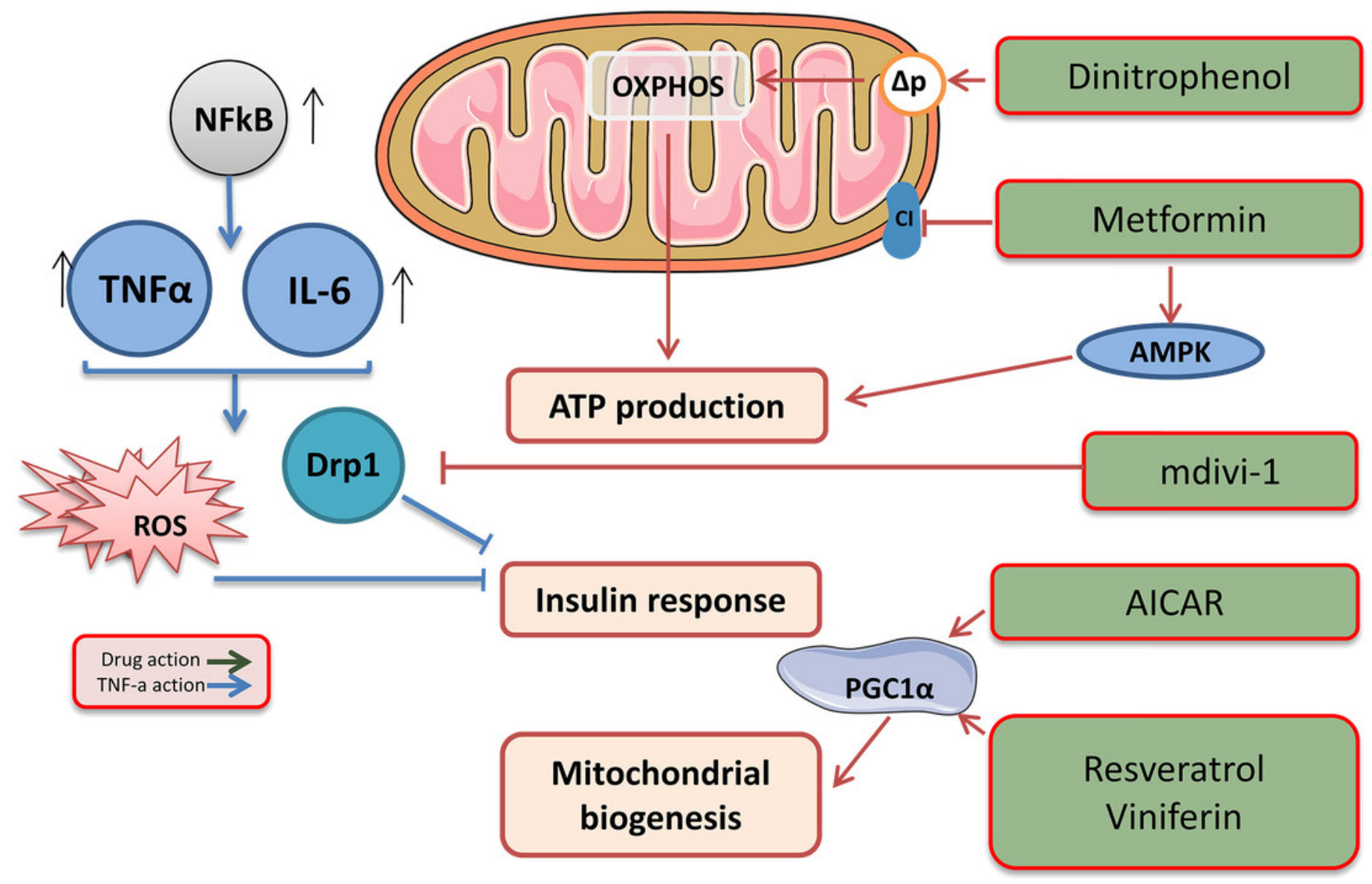

